\title{
Pharmacokinetic properties and antitumor efficacy of the 5-fluorouracil loaded PEG-hydrogel
}

\author{
Hee Yi1, Hee-Jung Cho', Soo-Min Cho', Dong-Goo Lee', AM Abd El-Aty'1,6, So-Jeong Yoon², Gun-Won Bae², \\ Kwang Nho², Bokyung Kim³ ${ }^{3}$ Chi-Ho Lee ${ }^{4}$, Jin-Suk Kim¹, Michael G Bartlett ${ }^{5}$ and Ho-Chul Shin*1
}

\begin{abstract}
Background: We have studied the in vitro and in vivo utility of polyethylene glycol (PEG)-hydrogels for the development of an anticancer drug 5-fluorouracil (5-FU) delivery system.

Methods: A 5-FU-loaded PEG-hydrogel was implanted subcutaneously to evaluate the drug retention time and the anticancer effect. For the pharmacokinetic study, two groups of male rats were administered either an aqueous solution of 5-FU (control group)/or a 5-FU-loaded PEG-hydrogel (treated group) at a dose of $100 \mathrm{mg} / \mathrm{kg}$. For the pharmacodynamic study, a human non-small-cell lung adenocarcinoma (NSCLC) cell line, A549 was inoculated to male nude mice with a cell density of $3 \times 10^{6}$. Once tumors start growing, the mice were injected with 5-FU/or 5-FU-loaded PEG-hydrogel once a week for 4 weeks. The growth of the tumors was monitored by measuring the tumor volume and calculating the tumor inhibition rate (IR) over the duration of the study.

Results: In the pharmacokinetic study, the 5-FU-loaded PEG-hydrogel gave a mean residence time (MRT) of $8.0 \mathrm{~h}$ and the elimination half-life of $0.9 \mathrm{~h}$; these values were 14- and 6 -fold, respectively, longer than those for the free solution of 5 -FU ( $p<0.05)$. In the pharmacodynamic study, A549 tumor growth was significantly inhibited in the 5-FU-loaded PEGhydrogel group in comparison to the untreated group beginning on Day $14(p<0.05-0.01)$. Moreover, the 5-FU-loaded PEG-hydrogel group had a significantly enhanced tumor IR $(p<0.05)$ compared to the free 5 -FU drug treatment group.

Conclusion: We suggest that 5-FU-loaded PEG-hydrogels could provide a useful tool for the development of an anticancer drug delivery system.
\end{abstract}

\section{Background}

The drug 5-Fluorouracil (5-FU) is one of the most common chemotherapeutic agents used against malignant tumors [1]. However, this drug has some pharmacokinetic limitations including unfavorable maximum drug concentrations (Cmax) and short half lives following systemic bolus injection. Earlier reports have demonstrated that acute increases in plasma 5-FU concentration can cause severe side effects and the antitumor effects of 5FU depend on exposure duration rather than plasma concentration levels [2]. Therefore, 5-FU acts more in a timedependent manner than in a dose-dependent manner [35]. Therefore, a continuous infusion system for the maintenance of intended levels may be beneficial. However, this approach might be unfavorable due to its high cost

* Correspondence: hshin@konkuk.ac.kr

1 Department of Veterinary Pharmacology and Toxicology, Konkuk University, Seoul 143-701, Republic of Korea

Full list of author information is available at the end of the article and patient compliance with long-term regimens. Implantable release devices have been attempted in vivo to reduce the period of hospitalization and eliminate the need for indwelling catheters [6]. Recently, we have developed PEG-hydrogel derivatives as an injectable sustained release device which can be bolused subcutaneously without any surgical implantation (US Patent 6858736; Korean Patent KR 2002-0089772 and 10-2004-0040782). We have hypothesized that this approach can provide a diffusional barrier for drug release and thereby deliver drugs for an extended period of time. In the current study, we have evaluated the drug release profiles of a 5FU-loaded PEG-hydrogel system. To confirm the therapeutic efficacy of the designed PEG controlled-release system, we conducted a pharmacodynamic study using the A549 tumor xenograft model in nude mice.

() 2010 Yi et al; licensee BioMed Central Ltd. This is an Open Access article distributed under the terms of the Creative Commons Attri:HoWed Central bution License (http://creativecommons.org/licenses/by/2.0), which permits unrestricted use, distribution, and reproduction in any medium, provided the original work is properly cited. 


\section{Methods}

\section{Chemicals and cell culture materials}

The 6-arm PEG-SG (6-arm polyethylene glycol Nhydroxy succinimidyl glutarate), and 6-arm PEG-AM (6arm polyethylene glycol amine) were developed by SunBio Inc. (An-yang, Republic of Korea). The 5-fluorouracil (5-FU), $\mathrm{NaH}_{2} \mathrm{PO}_{4}, \mathrm{Na}_{2} \mathrm{HPO}$, methyl alcohol (analytical grade), and diethyl ether were supplied by Sigma (St. Louis, MO, USA). Dulbecco's modified Eagle medium (DMEM), penicillin/streptomycin, and trypsin EDTA were purchased from GIBCO (Carlsbad, CA, USA). Aqueous fetal bovine serum (FBS) was obtained from WelGENE (Daegu, Republic of Korea).

\section{Cell Line and Animals}

For the pharmacokinetic study, male Sprague-Dawley rats were obtained from Orient Bio Inc. (Seongnam, Republic of Korea). The A549 human lung carcinoma cell line, a generous gift from Cha Biomedical Center (Seoul, Republic of Korea), was carefully inoculated into the dorsal neck of male nude mice (Balb/c Slc-nu/nu), which were supplied from the Central Lab Animal Inc. (Seoul, Republic of Korea). All in vivo experiments were carried out with the approval of the Institutional Animal Care and Use Committee (IACUC) at Konkuk University and in harmony with the Guide for Laboratory Animal Care and Use (IACUC Approval No. KUV7015, 7016)

\section{Preparation of 5-FU-loaded PEG hydrogel and in vitro Release of 5-FU-loaded PEG-hydrogel}

The 5-FU-loaded PEG-hydrogel was designed as shown in Fig 1. The 6-arm PEG-AM was dissolved in $10 \mathrm{mM}$ phosphate buffer ( $\mathrm{pH} 6.0$ ), and 5-FU was added into the solution at a concentration of $1 \mathrm{mg} / \mathrm{ml}$. The 6 -arm PEGSG was also dissolved in $10 \mathrm{mM}$ phosphate buffer $(\mathrm{pH}$ 6.0). The 6-arm PEG-AM and 6-arm PEG-SG solutions were prepared at various concentrations (i.e., 7, 10, and $15 \% \mathrm{w} / \mathrm{v})$. Approximately, $0.5 \mathrm{ml}$ of 6 -arm PEG-AM solution containing 5 -FU was placed in a 6-well plate, and an equal volume of 6 -arm PEG-SG solution $(0.5 \mathrm{ml})$ was added and mixed. The plate was shaken vigorously until the solutions hardened, forming the hydrogel. After hardening, the hydrogel was washed gently with $1 \mathrm{ml}$ of 10 $\mathrm{mM}$ phosphate buffer ( $\mathrm{pH} 7.2)$ to remove the any residual 5 -FU left on the hydrogel surface. The in vitro release study was carried out over 8 days. To quantify 5-FU released from the hydrogel, $2.5 \mathrm{ml}$ of $10 \mathrm{mM}$ phosphate buffer ( $\mathrm{pH}$ 7.2) was added into each well and replaced every day. The plate was sealed and placed at room temperature. The quantity of 5-FU released from the hydrogel was measured by monitoring the absorbance at 265 $\mathrm{nm}$. Since the released NHS or other PEG compounds gave additional signals at this wavelength, we carried out an additional experiment to assess background values without 5-FU. We calculated the net concentration of 5-
FU by subtracting the background signals resulting from other compounds from the total signal.

Fig. 1 shows the cross-linking reaction of 6-arm PEGamine (PEG-AM) and 6-arm PEG-succinimidyl glutarate (PEG-SG). When the PEG-SG is mixed with PEG-AM, PEG-SG separates the N-hydroxy succinimides (NHS) from its arms. Additionally, the terminals of PEG-SG ($\left.\mathrm{CO}_{2}\right)$ are cross-linked with the amine $\left(-\mathrm{NH}_{2}\right)$ groups of PEG-AM. This cross-linking between PEG-AM and PEG-SG changes the two PEG solutions into a gelatinous form (US Patent 6858736).

\section{Pharmacokinetics}

Rats were divided into two groups: a free 5-FU treatment group and a 5-FU-loaded PEG-hydrogel group. An aqueous solution of free 5-FU and a 5-FU-loaded PEG-hydrogel were administered subcutaneously to the rats at a dose of $100 \mathrm{mg} / \mathrm{kg}$. To formulate the 5-FU-loaded PEGhydrogel, PEG-SG and PEG-AM containing 5-FU were dissolved in sodium phosphate buffer ( $\mathrm{pH} 8.0)$ and mixed together in equal volumes. The free 5-FU drug solution was injected into rats using a normal syringe, and the 5FU-loaded PEG-hydrogel was injected using a mixing syringe device (Doowon Meditec Corp., Youngin-city, Republic of Korea). The aqueous solution of PEG-hydrogel (PEG-SG and PEG-AM) immediately changed into a gel after passing through the injection needle. Blood samples were collected at minutes $(0,5$ and 30$)$ and hours (1, 2, 3, 4, 12, and 24) after drug administration. Sampling continued until the PEG-hydrogel could not be palpated under the skin. Blood samples were centrifuged at 8000 rpm for 5 minutes (HANIL Science Industrial Co., Inchon, Republic of Korea), and harvested serum (150$200 \mu \mathrm{l})$ was subjected to HPLC analysis [7]. Samples were extracted using ethyl acetate $(6 \sim 8 \mathrm{ml})$ and then evaporated to dryness under $\mathrm{N}_{2}$ gas in a water bath adjusted at $60^{\circ} \mathrm{C}$. A $1.5 \mathrm{mM}$ sodium phosphate buffer ( $\mathrm{pH} 5.8$ ) was added to reconstitute the residue. Approximately 200-300 $\mu \mathrm{l}$ of the reconstituted solutions were filtered and injected for HPLC (Agilent 1100, Santa Clara, CA, USA). Separation was accomplished via isocratic elution of the mobile phase, which contained methanol and $1.5 \mathrm{mM}$ sodium phosphate buffer (99:1, v/v, pH 5.8), with a flow rate of $1 \mathrm{ml} / \mathrm{min}$. A $\mathrm{C}_{18}$ (Capcell Pak UG120, $5 \mu \mathrm{m}, 4.6 \mu \mathrm{m}$ I.D. $\times 250 \mu \mathrm{m}$, Shiseido, Tokyo, Japan) was used as an analytical column. The analysis was carried out at a column temperature of $40^{\circ} \mathrm{C}$. The wavelengths of the FLD (fluorescence detector) were $260 \mathrm{~nm}$ and $350 \mathrm{~nm}$ for excitation and emission, respectively.

Pharmacokinetic parameters were estimated for each rat by using the program "WinNonlin" to fit the serum concentration versus time data to the following equation: $C_{p}=\left(K_{a}^{*} F^{*} D\right) /\left\{V_{d}^{*}\left(k_{a}-k_{e}\right)\right\}^{*}\left(e x p^{-k e l^{*} t}-e x p^{-k a^{*} t}\right)$, where $C_{p}$ is the serum concentration and $\mathrm{k}_{\mathrm{a}}$ and $\mathrm{k}_{\mathrm{el}}$ are the absorption 


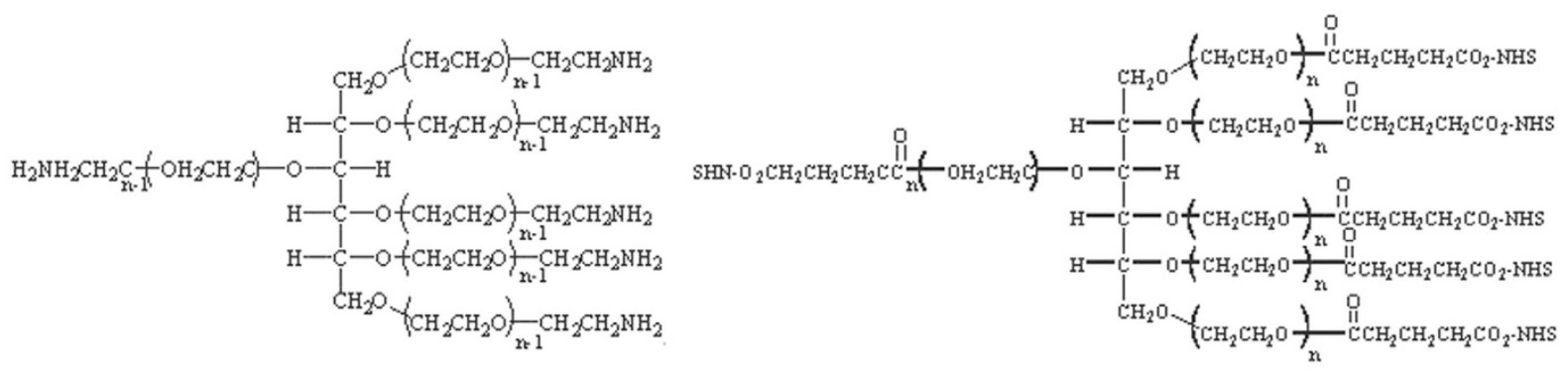

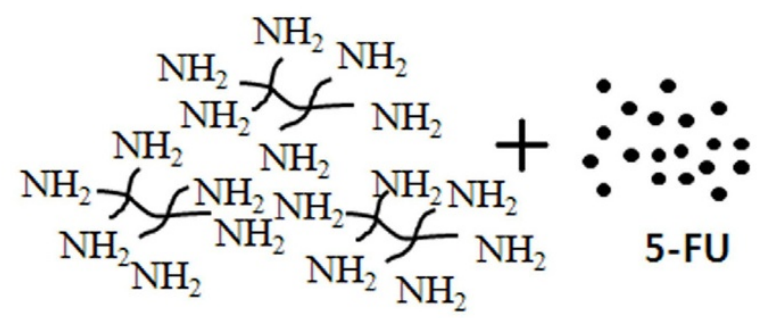

6-arm PEG-AM

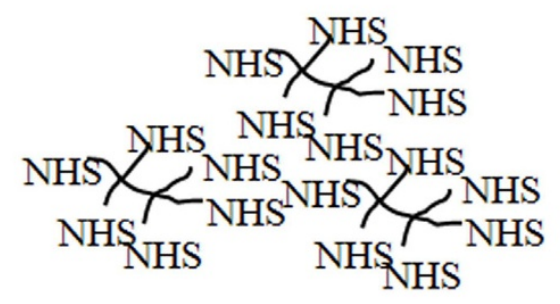

6-arm PEG-SG

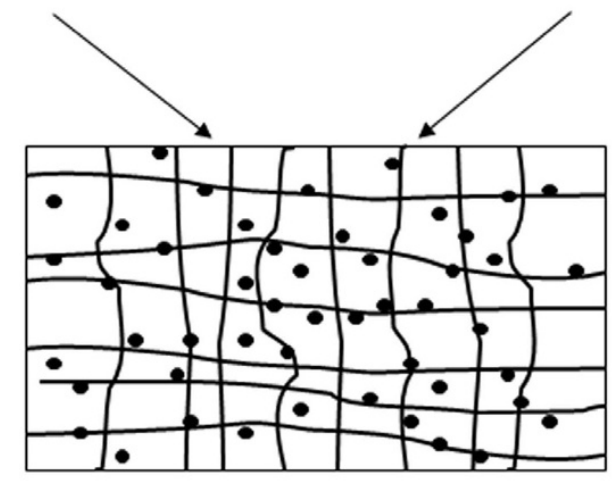

\section{Hydrogel network containing drug}

Figure 1 The chemical structures of the 6-arm PEG- AM and 6-arm PEG-SG. PEG-hydrogel network made by 6-arm PEG-AM and 6-arm PEG-SG. After displacement of the NHS (N-hydroxy succinimide) from 6-arm PEG-SG, amine residues on 6-arm PEG-AM are cross-linked with 6-arm PEG-SG.

and the elimination rate constants, respectively. The F, D, and $\mathrm{V}_{\mathrm{d}}$ represent the bioavailability, dose, and volume of distribution, respectively. The lag time was not considered, and the absorption and elimination rates were applied using first-order kinetics. The area under the concentration vursus time curve (AUC ${ }^{0-8}$ day) was calculated using the trapezoidal rule from time $t=0$ to the last measured concentration on Day 8. Serum drug concentrations and the estimated pharmacokinetic parameters were reported as means \pm SD.

\section{Antitumor Activity of 5-FU-loaded PEG-hydrogels in Tumor-bearing Nude Mice}

A total of $3 \times 10^{6}$ of cells from the A549 line, a human non-small-cell lung adenocarcinoma cell line, were inoculated into the dorsal neck of 4-week-old male nude mice (Balb/c Slc-nu/nu) as described in earlier reports $[7,8]$.
The tumor mass was monitored every week. When the tumors grew to $100-400 \mathrm{~mm}^{3}$, the mice were randomized into three groups: an untreated control group, a free 5-FU drug control group, and a 5-FU-loaded PEG-hydrogel group. The free 5-FU drug and 5-FU-loaded PEG-hydrogel were prepared as described above was subcutaneously injecting around the tumor mass once per week for 4 weeks at a dose of $85 \mathrm{mg} / \mathrm{kg}$. Body weight, tumor volume, and clinical assessment of the mice were monitored every week until the end of the study. There was no significant difference in body weight changes among the groups. When tumors were palpable and visible, the tumor volume (TV) was measured with a Vernier caliper and calculated using the following formula:

$$
\operatorname{TV}\left(\mathrm{mm}^{3}\right)=1 / 2 \times \text { Length } \times \text { Width }^{2}
$$


The antitumor effect was estimated by calculating the relative tumor volume and the relative tumor inhibition rate (IR, \%). The relative tumor volume (RTV) represents the tumor volume when the drugs are given to the mice.

$$
\mathrm{RTV}(\text { Relative tumor volume })=\mathrm{V}_{\mathrm{t}} / \mathrm{V}_{0}
$$

where $V_{0}$ is the tumor volume when the drugs are given to the mice and $V_{t}$ is the tumor volume at each measurement.

$$
\operatorname{IR}(\text { Inhibition Rate })=\left(1-\mathrm{T}_{\mathrm{RTV}} / \mathrm{C}_{\mathrm{RTV}}\right) \times 100
$$

The $\mathrm{T}_{\mathrm{RTV}}$ represents the RTV of treated groups, and the $\mathrm{C}_{\mathrm{RTV}}$ represents the RTV of the untreated control group.

\section{Histopathological Examination}

Sections of A549 taken from subcutaneously transplanted tumor masses were fixed with formalin and embedded in paraffin. Five micrometer tissue sections were prepared and stained with $\mathrm{H} \& \mathrm{E}$.

\section{Statistical Analysis}

Multiple comparison tests for different treatment groups were conducted. An ANOVA multiple comparison test (Dunnett's test) was performed to determine which pairs of groups differed significantly. An unpaired Student's $t$ test was also used to compare the 5-FU-loaded PEGhydrogel group versus the 5-FU-treated control group. The level of significance was taken as $\mathrm{p}<0.05$ or 0.01 . Values represent means \pm SD. Statistical analyses were performed with Statistical Analysis Systems software (SAS/STAT Version 8.1, Cary, NC, USA).

\section{Results}

\section{In vitro Release of 5-FU from PEG-hydrogel}

The optimal cross-linking reaction was obtained using 1 $\mathrm{mM}$ phosphate buffer ( $\mathrm{pH}$ of 8.0 ). Fig. 2 presents the cumulative releases of 5-FU from the 5-FU-loaded PEGhydrogel at 7, 10, and 15\% PEG concentrations. The $15 \%$ PEG-hydrogel released 5-FU much more slowly than did the $7 \%$ and $10 \%$ PEG-hydrogels. Most of the 5 -FU in the $7 \%$ and $10 \%$ of PEG-hydrogels was released within 2 days, whereas the 15\% PEG-hydrogel released 5-FU over a period of 5 days.

\section{Pharmacokinetics of the Free 5-FU Drug and 5-FU- loadedPEG-hydrogel}

Fig. 3 shows the average serum concentration versus time curves of 5-FU following subcutaneous administration of both the free 5-FU drug and 5-FU-loaded PEG-hydrogel to rats at $100 \mathrm{mg} / \mathrm{kg}$. The 5-FU-loaded PEG-hydrogel released 5-FU into the serum over a period of one week, whereas the 5-FU from the free drug injection rapidly disappeared from the serum within several hours after injection. The serum concentration profiles for the free 5FU and 5-FU-loaded PEG-hydrogel were well described by a one-compartment open pharmacokinetic model. As shown in Table 1, between the two groups there were marked differences in some parameters including the maximum serum concentrations $\left(C_{\max }\right)$, the elimination half-lives $\left(t_{1 / 2}\right)$ and the area under the curves (AUC). The $\mathrm{C}_{\max }$ and $\mathrm{t}_{1 / 2}$ in the free 5 -FU treated group were about 68 $\mu \mathrm{g} / \mathrm{ml}$ and $0.15 \mathrm{~h}$, respectively, while those parameters in 5-FU-loaded PEG-hydrogel group were $8 \mu \mathrm{g} / \mathrm{ml}$ and 0.9 $\mathrm{h}$, respectively. In the free 5 -FU treated group, the AUC and the area under the moment curve (AUMC) were roughly $60 \mu \mathrm{g} \mathrm{h} / \mathrm{ml}$ and $33 \mu \mathrm{g} \mathrm{h} / \mathrm{ml}$, respectively. In the 5-FU-loaded PEG-hydrogel group, the AUC and AUMC were $14 \mu \mathrm{g} \mathrm{hr} / \mathrm{ml}$ and $112 \mu \mathrm{g} \mathrm{h} / 2 \mathrm{ml}$, respectively.

\section{Tumorigenicity of the A549 Xenograft and Histopathology}

A total of $3 \times 10^{6}$ A549 cells were inoculated into nude mice. The subcutaneous inoculation of tumor cells resulted in tumor generation at the injection site. Solid tumors were locally measurable by 1 month after inoculation. As shown in Fig. 4, the relative tumor volume (RTV) of the untreated control group markedly increased in a time-dependent manner. However, the RTVs were significantly suppressed compared to the untreated group by 5 FU-loaded PEG-hydrogel treatment starting on Day 14. However, the free 5-FU drug also showed significant inhibition of tumor volume increases on Day 28. The histopathological examination revealed typical adenocarcinoma patterns, including the mixed acinar and tubular form characterized by acini and tubules composed of cuboidal and columnar cells. Poorly differentiated tumor cells are shown among the cells. Some cancer cells infiltrated into the muscular layer.

\section{Comparative Antitumor Effects of the Free 5-FU and 5-FU loaded PEG-hydrogel}

Compared to the free 5-FU-treated group, A549 tumor growth was significantly inhibited in the 5-FU-loaded PEG-hydrogel group on Day 28 ( $\mathrm{p}<0.05$ ). The 5-FUloaded PEG-hydrogel produced an inhibition rate of $64 \%$, and the free 5-FU drug produced an inhibition rate of $38 \%$. The PEG-hydrogel itself showed no cytotoxicity for A549 cells and had no inhibitory effect on tumor growth in the mouse xenograft (data not shown).

\section{Discussion}

Because the use of 5-FU is limited by its short half-life and rapid elimination, short duration 5-FU bolus administration has a relatively low response rate [9]. For these reasons, suitable methods for the administration of 5-FU have been investigated for a long time [10-12]. Since continuous intravenous infusion has the advantage of maintaining a low concentration of the drug in the blood for 


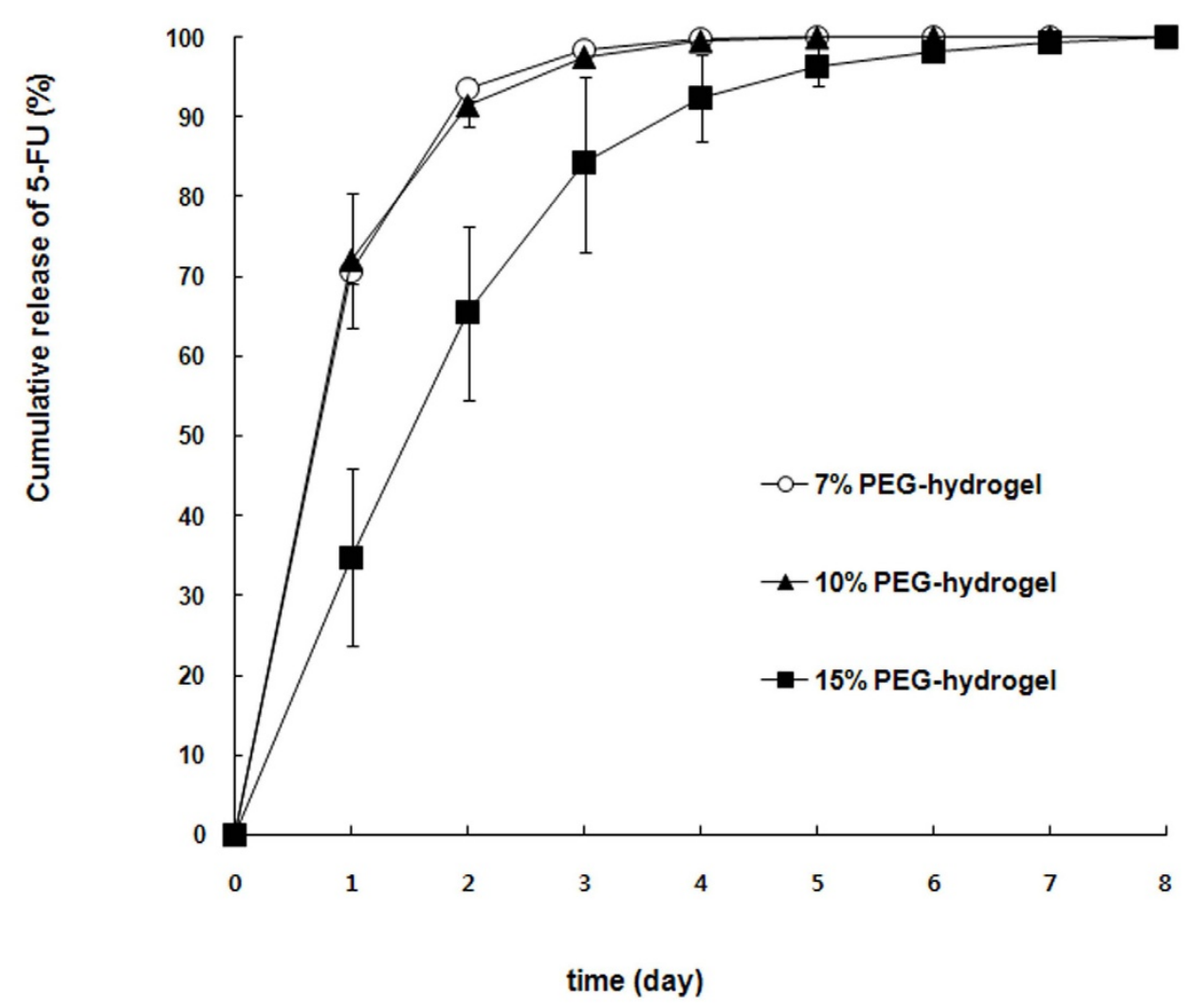

Figure $\mathbf{2}$ In vitro release of 5-FU from PEG-hydrogel. The error bars represent the range from two experiments.

an extended period of time, continuous 5-FU infusion has been more effective than bolus administration in clinical trials $[13,14]$. In comparison to bolus administration, continuous infusion also minimizes the severe, lifethreatening toxic effects of 5-FU [15,16]. Accordingly, continuous infusion is an effective method for both increasing the length of time for which the drug contacts tumor cells and reducing toxicity. However, continuous infusion may cause patient discomfort due to indwelling catheters and bed restriction, and it may give rise to complications like catheter-related vasculitis and sepsis $[15,17]$. Therefore, controlled-release devices (e.g., biodegradable polymers) have been previously studied for the development of anticancer drug delivery $[18,19]$.

An optimal crosslinking reaction of PEG-SG and PEGAM was observed in $1 \mathrm{mM}$ phosphate buffer at a $\mathrm{pH}$ of 8.0. PEG-hydrogel formation was maximized by using $15 \%$ concentrations of PEG-AM and PEG-SG. These conditions of PEG-hydrogel formation were applied in our in vivo pharmacokinetics and antitumor screening studies. Furthermore, this PEG-hydrogel system is based on a very simple injection that does not require a surgical procedure.

Many other groups have reported that the pharmacokinetics of 5-FU in patients vary significantly based on the dosing regimen [20]. In addition, like other anticancer chemotherapeutics, 5-FU has a relatively narrow therapeutic index [21]. For these reasons, controlling the serum concentration of the drug is important for drug efficacy and safety. When injected subcutaneously in our study, 5-FU disappeared rapidly from systemic circulation. On the other hand, the elimination of 5-FU from the 5-FU-loaded PEG-hydrogel showed significantly delayed release from systemic circulation. The mean residence time of 8 hours is approximately 14 times higher than that of free 5-FU administered to animals. Moreover, the elimination half-life of the 5-FU-loaded PEG-hydrogel was $0.9 \mathrm{~h}$; this is 6-fold larger than that of the free 5-FUtreated control group. Blanco et al. [22] also evaluated the drug release profile of a subcutaneously implanted poly (2-hydroxyethylmethacrylate-co-acrylamide) hydrogel containing 5-FU. In this study, the drug was released 


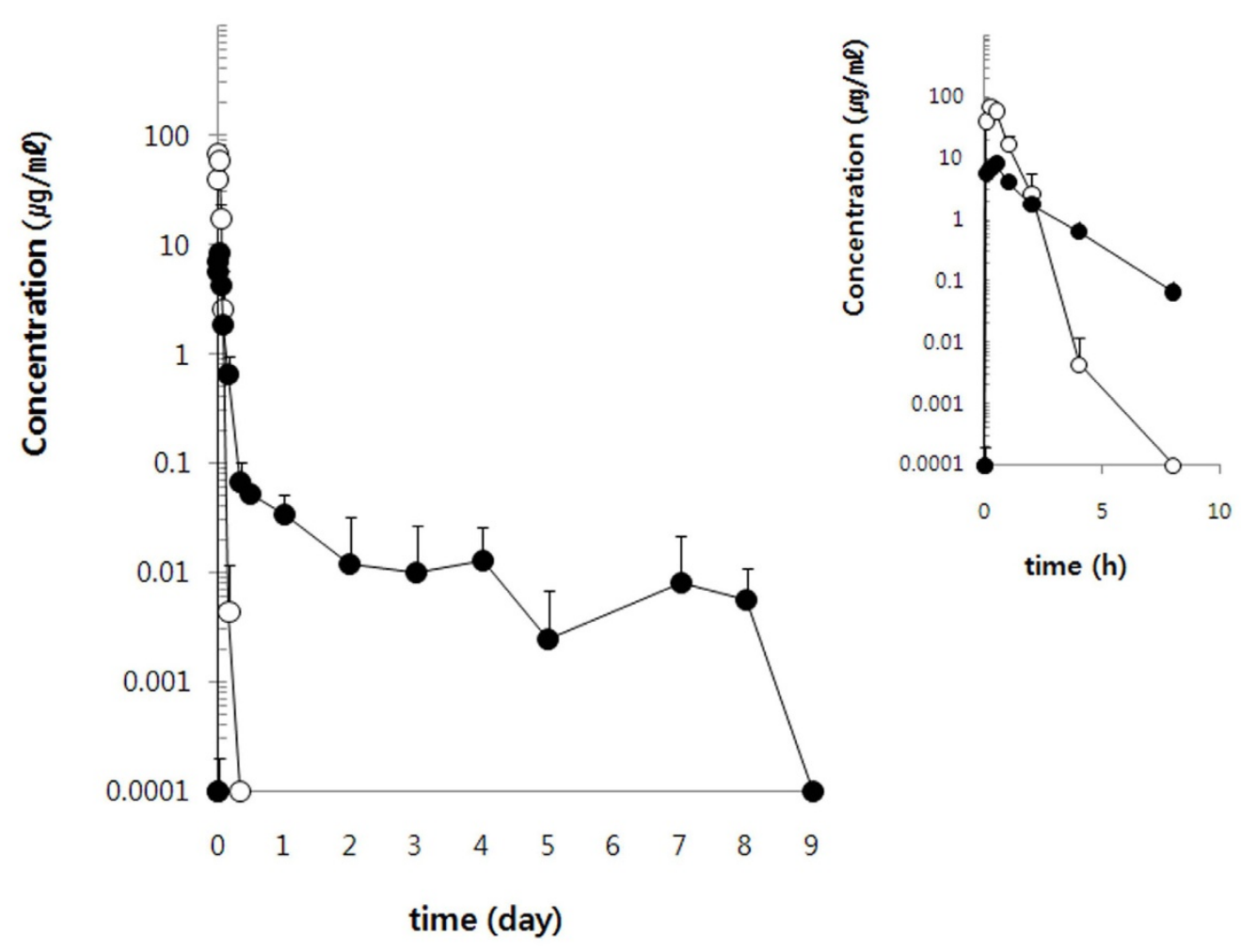

Figure 3 Serum concentration curves for 5 -FU following subcutaneous injection of $100 \mathrm{mg} / \mathrm{kg}$ of the free 5 -FU drug (open circle) and 5-FUloaded PEG-hydrogel (closed circle) to SD rats $(\mathbf{n}=\mathbf{3})$. Right panel shows serum concentration curves through 0 to 8 hour.

from the poly hydrogel over a span of 2 days. In our study, the PEG-hydrogel released 5-FU more than 4 days after the injection. Although Blanco et al. [22] employed a surgical incision for the implantation of the poly hydrogel, we implanted the hydrogel by direct injection using a mixing syringe device in which the SG and AM solutions were immediately changed into a solid gel by mixing together.

We report that the controlled release of 5-FU from by the PEG-hydrogel effectively suppressed tumor growth in vivo. Compared to the free 5-FU-treated control group, the 5-FU-loaded PEG-hydrogel group demonstrated a

Table 1: Pharmacokinetic parameters of 5-FU after the subcutaneous injection of $100 \mathrm{mg} / \mathrm{kg}$ of the free 5-FU drug and 5FU-loaded PEG-hydrogel into SD rats.

\begin{tabular}{lllc}
\hline Parameters & unit & $\begin{array}{l}\mathbf{5 - F U} \\
(\mathbf{n}=\mathbf{3}, \mathbf{M e a n} \pm \mathbf{S D})\end{array}$ & $\begin{array}{c}\text { 5-FU-loaded PEG-hydrogel } \\
\text { (n= 3, Mean } \pm \text { SD) }\end{array}$ \\
\hline $\mathrm{V}_{\mathrm{d}} / \mathrm{F}$ & & & $10.60 \pm 1.55$ \\
$\mathrm{t}_{1 / 2(a)}$ & $\mathrm{L}$ & $0.41 \pm 0.04$ & $0.06 \pm 0.03$ \\
$\mathrm{t}_{1 / 2}$ & $\mathrm{~h}$ & $0.21 \pm 0.06$ & $0.87 \pm 0.17^{*}$ \\
$\mathrm{CL}_{\text {tot }} / \mathrm{F}$ & $\mathrm{h}$ & $0.15 \pm 0.04$ & $8.46 \pm 1.07$ \\
$\mathrm{C}_{\max }$ & $\mathrm{l} / \mathrm{h}$ & $2.04 \pm 0.51$ & $7.47 \pm 1.63$ \\
$\mathrm{~T}_{\max }$ & $\mu \mathrm{g} / \mathrm{ml}$ & $67.81 \pm 15.84$ & $0.33 \pm 0.14$ \\
$\mathrm{AUC}(0-8$ day & $\mathrm{h}$ & $0.25 \pm 0.00$ & $14.14 \pm 1.20$ \\
$\mathrm{MRT}$ & $\mu \mathrm{g} \cdot \mathrm{h} / \mathrm{ml}$ & $59.71 \pm 10.58$ & $8.03 \pm 4.41^{*}$
\end{tabular}

$\mathrm{V}_{\mathrm{d}} / \mathrm{F}$; apparent volume of distribution, $\mathrm{t}_{1 / 2(\mathrm{a})} ;$ absorption half life, $\mathrm{t}_{1 / 2}$; elimination half life, $\mathrm{CL}_{\text {tot }} / \mathrm{F} ;$ total body clearance, $\mathrm{C}_{\text {max }} ;$ maximum serum concentration, $T_{\text {maxi }}$ time to maximum concentration, AUC; area under the curve, MRT; mean residence time (AUMC/AUC). ${ }^{*} 5$-FU vs. 5-FUloaded PEG-hydrogel $(p<0.05)$. 


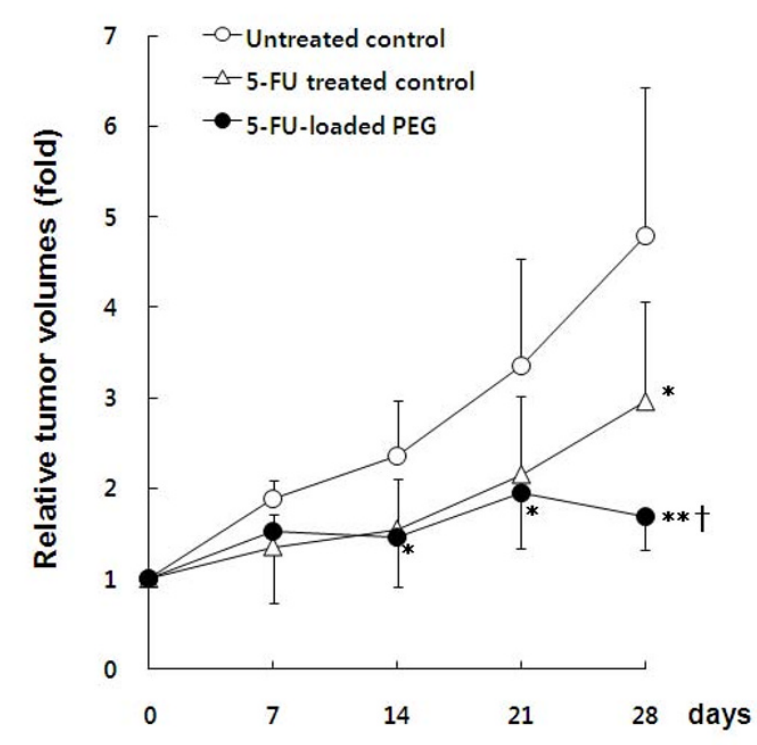

Figure 4 Comparison of relative tumor volumes among treatment groups. * Compared to the untreated control group $\left(n=6,{ }^{*} p\right.$ $\left.<0.05,{ }^{* *} p<0.01\right) .{ }^{+}$Compared between 5-FU-treated control and 5FU-loaded PEG-hydrogel groups $(n=6,+p<0.05)$.

strong tumor growth inhibition effect. Following injection once a week, the tumor inhibition rate (IR) of the 5FU-loaded PEG-hydrogel animals markedly increased from 20\% (Day 7) to 65\% (Day 28). In contrast, the IR of animals in the free 5-FU-treated control group showed a smaller increase from 30 to $40 \%$. These pharmacodynamic data demonstrate that the PEG-hydrogel system is very effective in maintaining the optimal blood concentration of 5-FU necessary to suppress growth of tumor cells efficiently. The current finding is in agreement with the fact that 5-FU is not a dose-dependent but rather a time-dependent drug [3-5].

The common acute toxic effects of 5-FU include myelosuppression, mucositis, and diarrhea $[15,23,24]$. It is generally understood that the toxicity of $5-\mathrm{FU}$ is related to the AUC $[20,25,26]$. In our pharmacokinetic studies, the AUC and $\mathrm{C}_{\max }$ of 5-FU were markedly decreased after treatment with the PEG-hydrogel system. These findings may suggest that 5-FU treatment with a PEG-hydrogel may be used to reduce the severe toxic effects of this drug.

\section{Conclusions}

Our results suggest that the injectable PEG-hydrogel system offers an efficient approach to cancer therapy using a direct injection method that circumvents surgical incision.

\section{Abbreviations}

(PEG): polyethylene glycol; (5-FU): 5-fluorouracil; (IR): inhibition rate; (MRT): mean residence time; (PEG-SG,): polyethylene glycol N-hydroxy succinimidyl glutarate; (PEG-AM): polyethylene glycol amine; (TV): tumor volume; (RTV): relative tumor volume; $\left(C_{\text {max }}\right)$ : maximum serum concentration; $\left(T_{\max }\right)$ : time to maximum concentration; $\left(\mathrm{t}_{1 / 2}\right)$ : elimination half-life; (AUC): area under the curve; (AUMC): area under the moment curve.

\section{Competing interests}

The authors declare that they have no competing interests.

\section{Authors' contributions}

$\mathrm{HY}, \mathrm{KN}$ and $\mathrm{HS}$ conceived the study and finalized the manuscript. HY, HC, SC and DL carried out the sample preparation, pharmacokinetics, histopathological examination and in vivo and vitro antitumor studies. SY, GB and KN developed the 6-arm PEG-SG and 6-arm PEG-AM, and performed in vitro release study of 5-FU from PEG-hydrogel. AMAE, BK, CL, MGB and JK contributed to the scientific discussion and the manuscript editing. All authors read and approved the final manuscript.

\section{Acknowledgements}

This work was supported by the Konkuk University in 2009

\section{Author Details}

1Department of Veterinary Pharmacology and Toxicology, Konkuk University, Seoul 143-701, Republic of Korea, 2SunBio Inc, Anyang 431-804, Republic of Korea, ${ }^{3}$ Department of Physiology, School of Medicine, Konkuk University, Seoul 143-701, Republic of Korea, ${ }^{4}$ Department of Food Science and Biotechnology of Animal Resources, Konkuk University, Seoul 143-701, Republic of Korea, ${ }^{5}$ Department of Pharmaceutical and Biomedical Sciences, University of Georgia, Athens, GA 30602, USA and ${ }^{6}$ Current address: Department of Pharmacology, Faculty of Veterinary Medicine, Cairo University, Giza, Egypt

Received: 11 September 2009 Accepted: 18 May 2010

Published: 18 May 2010

\section{References}

1. Heidelberger C, Chaudhuri NK, Danneberg P, Mooren D, Griesbach L, Duschinsky R, Schnitzer RJ, Pleven E, Scheiner J: Fluorinated pyrimidines, a new class of tumour-inhibitory compounds. Nature 1957, 179:663-666.

2. Tanaka F, Fukuse T, Wada H, Fukushima M: The history, mechanism and clinical use of oral 5-fluorouracil derivative chemotherapeutic agents. Curr Pharm Biotechnol 2000, 1:137-164.

3. Calabro-Jones PM, Byfield JE, Ward JF, Sharp TR: Time-dose relationships for 5 -fluorouracil cytotoxicity against human epithelial cancer cells in vitro. Cancer Res 1982, 42:4413-4420.

4. Drewinko B, Yang LY: Cellular basis for the inefficacy of 5-FU in human colon carcinoma. Cancer Treat Rep 1985, 69:1391-1398.

5. Link KH, Aigner KR, Peschau K, Warthona M, Schwemmle K, Danenberg PV: Concentration and time dependence of the toxicity of fluorinated pyrimidines to HT 29 colorectal carcinoma cells. Cancer Chemother Pharmacol 1988, 22:58-62.

6. Weissleder R, Poss K, Wilkinson R, Zhou C, Bogdanov A Jr: Quantitation of slow drug release from an implantable and degradable gentamicin conjugate by in vivo magnetic resonance imaging. Antimicrob Agents Chemother 1995, 39:839-845.

7. Fabbri M, Iliopoulos D, Trapasso F, Aqeilan Rl, Cimmino A, Zanesi N, Yendamuri S, Han SY, Amadori D, Huebner K, Croce CM: WWOX gene restoration prevents lung cancer growth in vitro and in vivo. Proc Natl Acad Sci USA 2005, 102:15611-15616.

8. Jiang Y, Cui L, Yie TA, Rom WN, Cheng H, Tchou-Wong KM: Inhibition of anchorage-independent growth and lung metastasis of A549 lung carcinoma cells by lkappaBbeta. Oncogene 2001, 20:2254-2263.

9. Kemeny $\mathrm{N}$ : Role of chemotherapy in the treatment of colorectal carcinoma. Semin Surg Oncol 1987, 3:190-214.

10. Drewinko B, Yang LY, Ho DH, Benvenuto J, Loo TL, Freireich EJ: Treatment of cultured human colon carcinoma cells with fluorinated pyrimidines. Cancer 1980, 45:1144-1158. 
11. Murnane JP, Byfield JE, Ward JF, Calabro-Jones P: Effects of methylated xanthines on mammalian cells treated with bifunctional alkylating agents. Nature 1980, 285:326-329.

12. Roper PR, Drewinko B: Comparison of in vitro methods to determine drug-induced cell lethality. Cancer Res 1976, 36:2182-2188.

13. Moertel CG, Schutt AJ, Reitemeier RJ, Hahn RG: A comparison of 5fluorouracil administered by slow infusion and rapid injection. Cancer Res 1972, 32:2717-2719.

14. Meta-analysis Group In Cancer: Efficacy of intravenous continuous infusion of fluorouracil compared with bolus administration in advanced colorectal cancer. Meta-analysis Group In Cancer. J Clin Oncol 1998, 16:301-308.

15. Hansen RM, Ryan L, Anderson T, Krzywda B, Quebbeman E, Benson A, Haller DG, Tormey DC: Phase III study of bolus versus infusion fluorouracil with or without cisplatin in advanced colorectal cancer. J Natl Cancer Inst 1996, 88:668-674.

16. Seifert P, Baker LH, Reed ML, Vaitkevicius VK: Comparison of continuously infused 5-fluorouracil with bolus injection in treatment of patients with colorectal adenocarcinoma. Cancer 1975, 36:123-128.

17. Desai AA, Vogelzang NJ, Rini BI, Ansari R, Krauss S, Stadler WM: A high rate of venous thromboembolism in a multi-institutional phase II trial of weekly intravenous gemcitabine with continuous infusion fluorouracil and daily thalidomide in patients with metastatic renal cell carcinoma. Cancer 2002, 95:1629-1636.

18. Lalloo A, Chao P, Hu P, Stein S, Sinko PJ: Pharmacokinetic and pharmacodynamic evaluation of a novel in situ forming poly(ethylene glycol)-based hydrogel for the controlled delivery of the camptothecins. J Control Release 2006, 112:333-342.

19. Qiu B, Stefanos S, Ma J, Lalloo A, Perry BA, Leibowitz MJ, Sinko PJ, Stein S: A hydrogel prepared by in situ cross-linking of a thiol-containing poly(ethylene glycol)-based copolymer: a new biomaterial for protein drug delivery. Biomaterials 2003, 24:11-18.

20. Greenblatt MS, Mangalik A, Ferguson J, Elias L: Phase I evaluation of therapy with four schedules of 5 -fluorouracil by continuous infusion combined with recombinant interferon alpha. Clin Cancer Res 1995, 1:615-620.

21. Gamelin E, Boisdron-Celle M: Dose monitoring of 5-fluorouracil in patients with colorectal or head and neck cancer--status of the art. Crit Rev Oncol Hematol 1999, 30:71-79.

22. Blanco MD, Garcia O, Gomez C, Sastre RL, Teijon JM: In-vivo drug delivery of 5-fluorouracil using poly(2-hydroxyethyl methacrylate-coacrylamide) hydrogels. J Pharm Pharmacol 2000, 52:1319-1325

23. Reitemeier RJ, Moertel CG: Comparison of rapid and slow intravenous administration of 5-fluorouracil in treating patients with advanced carcinoma of the large intestine. Cancer Chemother Rep 1962, 25:87-89.

24. Ansfield F, Klotz J, Nealon T, Ramirez G, Minton J, Hill G, Wilson W, Davis H $\mathrm{Jr}$, Cornell G: A phase III study comparing the clinical utility of four regimens of 5-fluorouracil: a preliminary report. Cancer 1977, 39:34-40.

25. Santini J, Milano G, Thyss A, Renee N, Viens P, Ayela P, Schneider M, Demard F: 5-FU therapeutic monitoring with dose adjustment leads to an improved therapeutic index in head and neck cancer. Br J Cancer 1989, 59:287-290.

26. Thyss A, Milano G, Renee N, Vallicioni J, Schneider M, Demard F: Clinical pharmacokinetic study of 5-FU in continuous 5-day infusions for head and neck cancer. Cancer Chemother Pharmacol 1986, 16:64-66.

\section{Pre-publication history}

The pre-publication history for this paper can be accessed here: http://www.biomedcentral.com/1471-2407/10/211/prepub

doi: $10.1186 / 1471-2407-10-211$

Cite this article as: $Y$ i et al., Pharmacokinetic properties and antitumor efficacy of the 5-fluorouracil loaded PEG-hydrogel BMC Cancer 2010, 10:211 